\title{
Estudo Longitudinal do Desenvolvimento de Crianças Nascidas Pré-Termo no Primeiro Ano Pós-natal
}

\author{
Longitudinal Study of the Development of Children Born Preterm \\ in the First Year of Post-natal age
}

\author{
Fabíola Dantas Andréz Nobre, Ana Emília Vita Carvalho, \\ Francisco Eulógio Martinez \& Maria Beatriz Martins Linhares * \\ Universidade de São Paulo
}

\begin{abstract}
Resumo
O objetivo do estudo foi avaliar indicadores de desenvolvimento de crianças nascidas pré-termo e comparar o desempenho nas faixas de 5 a 7 e 10 a 14 meses de idade corrigida. Foram relacionados indicadores do desenvolvimento com variáveis neonatais e do ambiente familiar e sexo. Trinta crianças foram avaliadas pelo Denver-II. As cuidadoras responderam à entrevista e o prontuário médico foi analisado. Na faixa de 5 a 7 meses, $20 \%$ das crianças apresentaram risco para problemas de desenvolvimento e $27 \%$ de 10 a 14 meses. Houve diferença significativa entre as fases com mais risco na linguagem na faixa de 10 a 14 meses. As crianças nascidas com menor peso e idade gestacional, que permaneceram mais tempo hospitalizadas e com situações familiares adversas apresentaram mais problemas de desenvolvimento. Palavras-chave: Pré-termo; Muito baixo peso; Desenvolvimento; Saúde.
\end{abstract}

\begin{abstract}
The aim of the study was to assess the development of children born preterm and to compare the performance in the ranges of 5-7 and 10-14 months of corrected age. The children's development indicators and the variables of neonatal period and familial environment were correlated. Thirty children were assessed by Denver-II. The caregivers were interviewed and the medical chart was examined. Twenty per cent of children presented risk for developmental problems at 5-7 months, and 27\% at 10-14 months. There was statistical significant difference between ages in language, with high risk at 10-14. The children with lower birthweight, lower gestational age, longer time stay in the hospital, and with adverse family situations showed higher risk for developmental problems.

Keywords: Pre-term; Very low weight; Development; Health.
\end{abstract}

Na trajetória de desenvolvimento de crianças podem ocorrer adversidades que constituem-se em fatores de risco tornado-as vulneráveis para o enfrentamento das tarefas evolutivas. De acordo com Lewis, Dlugokinski, Caputo e Griffin (1988), os fatores de risco podem envolver a criança, a família nuclear e o ambiente ampliado, incluindo, portanto, tanto fatores biológicos quanto psicossociais. Entre os fatores biológicos podese destacar o nascimento pré-termo, que ocorre antes da $37^{\mathrm{a}}$ semana de gestação, com muito baixo peso (abaixo de 1.500 gramas).

O nascimento prematuro constitui-se em um grave problema na área de Saúde da Criança, considerando-se que

\footnotetext{
* Endereço para correspondência: Universidade de São Paulo, Faculdade de Medicina, Laboratório de Pesquisa Prevenção de Problemas de Desenvolvimento e Comportamento da Criança, Prédio da Saúde Mental - Avenida Tenente Catão Roxo, 2650, salas 52 e 53, Ribeirão Preto, SP, Brasil, CEP 14049-900. Tel.: (55) (16) 3602 4610; Fax: (55) (16) 3602 4504.E-mail: linhares@ fmrp.usp.br Agradecemos em especial às crianças e mães participantes do estudo, assim como ao apoio financeiro da Coordenação
}

a maior causa da mortalidade infantil no Brasil está associada às condições perinatais e que, além disso, as taxas de prematuridade têm aumentado ao longo do tempo (Silveira et al., 2008). Segundo esses autores, a prevalência de nascimento pré-termo variou de 3 a $15 \%$ entre os anos de 1978 a 2004, nas regiões Sudeste do país, e mostrou uma tendência crescente a partir da data de 1990. Os últimos dados disponíveis do Sistema Único de Saúde (SUS), que se referem ao Ano-Base de 2005, mostram um índice de $6,6 \%$ de nascimento pré-termo considerando os nascidos vivos no Brasil; este índice aumenta para 7,4 \% na Região Sudeste, 7,8 \% no Estado de São Paulo e 9,3\% na cidade de Ribeirão Preto (Ministério da Saúde, n.d.).

Paralelamente, o nascimento prematuro é também um problema na área do Desenvolvimento da Criança, na

de Aperfeiçoamento de Pessoal de Nível Superior (CAPES) concedido à primeira autora por meio da Bolsa de Mestrado do Programa de Pós-graduação em Psicologia da Faculdade de Filosofia, Ciências e Letras de Ribeirão Preto da Universidade de São Paulo (FFCLRP-USP), com a orientação da última autora e do $\mathrm{CNPq}$ 
medida em que, além de ser um risco para mortalidade e morbidade, constitui-se em um risco relativo para transtornos de desenvolvimento e comportamento. Crianças nascidas pré-termo apresentaram mais problemas na área cognitiva (Laucht, Esser, \& Schmidt, 2002), motora (Carvalho, Linhares, \& Martinez, 2001) e do comportamento (Linhares et al., 2004) em comparação com crianças nascidas a termo. Além disso, esses problemas podem surgir em diferentes fases, ou seja, na fase pré-escolar (Méio et al., 2004), escolar (Linhares et al., 2004) e na adolescência (Greenley, Taylor, Drotar, \& Minich, 2007). Quanto maior o risco clínico neonatal do bebê nas primeiras horas de vida, maior o risco de mortalidade e morbidade (Zardo \& Procianoy, 2003) e problemas de desenvolvimento (Bordin, 2005). Desta forma considera-se que a prematuridade e os fatores associados constituem-se em risco para a saúde e desenvolvimento adaptativo do indivíduo.

A somatória de riscos na vida de um indivíduo e a persistência destes aumentam sobremaneira as chances da criança apresentar problemas no desenvolvimento (Linhares, 2004). Por outro lado, há um interjogo entre fatores de riscos e mecanismos de proteção que pode atenuar os efeitos negativos do risco ao desenvolvimento da criança (Silk et al., 2007). Entre os mecanismos de proteção podem ser identificados recursos do indivíduo ou do seu contexto de desenvolvimento. Os estudos com delineamento longitudinal são, nesse caso, úteis para compreensão dos riscos e proteção, recursos e dificuldades no desenvolvimento desses bebês e para subsidiar intervenções essenciais preventivas ou terapêuticas.

Os testes de triagem ou rastreamento para problemas de desenvolvimento podem detectar sinais de risco relativo que requerem intervenções preventivas. De acordo com um recente estudo de revisão, o Teste de Triagem do Desenvolvimento de Denver-II destaca-se entre os instrumentos de triagem mais utilizados no Brasil na avaliação de bebês prematuros (Santos, Araújo, \& Porto, 2008). O Denver-II tem sido utilizado com amostras de crianças nascidas prematuras, com peso ao nascimento abaixo de $1.500 \mathrm{~g}$ e nível sócio-econômico baixo (Victora, Barros, Tomasi, \& Halpern, 1996), desnutridas (Fisberg et al., 1997) e em risco psicossocial (Santa Maria-Mengel \& Linhares, 2007).

Os primeiros anos de vida exercem papel relevante, pois as experiências iniciais têm impacto decisivo na estrutura do cérebro e nas capacidades do adulto (Couperus \& Nelson, 2006). Deste modo, acompanhar o desenvolvimento no primeiro ano de vida pós-natal é de extrema importância devido a esta ser a fase em que a criança apresenta maior plasticidade cerebral (Mustard, 2002).

Considerando-se o exposto, os estudos sobre indicadores de risco relativo presentes no desenvolvimento inicial de bebês vulneráveis nascidos prematuros devem ser conduzidos focalizando-se os fatores de risco clínico neonatal do bebê e os indicadores do ambiente familiar.
O presente estudo ${ }^{1}$ longitudinal-prospectivo teve, por objetivos: (a) avaliar indicadores de desenvolvimento de bebês nascidas pré-termo com muito baixo peso e comparar esses indicadores em duas fases ao longo do primeiro ano; (b) relacionar os indicadores do desenvolvimento das crianças e as variáveis neonatais, o sexo e variáveis do ambiente familiar.

\section{Método}

\section{Participantes}

A amostra foi composta por 30 bebês nascidos pré-termo (menos de 37 semanas de idade gestacional) com muito baixo peso $(<1.500 \mathrm{~g})$, de ambos os sexos, no Hospital das Clínicas da Faculdade de Medicina de Ribeirão Preto da Universidade de São Paulo (HCFMRP-USP) com história de internação na Unidade de Tratamento Intensivo Neonatal-UTIN. Os bebês freqüentavam regularmente o Programa de Seguimento Longitudinal do Desenvolvimento Psicológico de Prematuros nascidos no Hospital das Clínicas da FMRP-USP (PSL) (Linhares et al., 2004). Participaram também do estudo as respectivas mães dos bebês. A amostra foi extraída de uma amostra mais ampla 86 crianças do estudo de Nobre (2005), sendo selecionadas de acordo com o critério de apresentar duas avaliações no primeiro ano de vida. O estudo foi aprovado pelo Comitê de Ética em Pesquisa do HCFMRP (Processo HCRP ${ }^{\circ}$ 2075/2003) e as guardiãs legais das crianças assinaram um termo de consentimento livre e esclarecido para participar do estudo.

\section{Instrumentos e Materiais}

Foram utilizados os seguintes instrumentos e materiais: (a) Teste de Triagem do Desenvolvimento de Denver-II (Denver II - Screening Manual) (Frankenburg, Dodds, Archer, \& Bresnick, 1990), traduzido e adaptado por Figueiras, Pedromônico, Sales e Figueiras (2000) e Drachler, Marshall e Leite (2007). Consiste em um teste de rastreamento (screening) de risco para problemas de desenvolvimento em crianças na faixa etária de um mês a seis anos, incluindo quatro áreas: motora ampla, motora adaptativa, linguagem e habilidade pessoal/social. Os comportamentos das crianças são observados diretamente e os itens avaliados em Passou, Falhou, Recusa e Não houve oportunidade. Alguns itens podem ser avaliados por Anamnese, de acordo com o relato do cuidador. Considera-se Risco quando no mínimo há dois Cuidados ou um Atraso em algum item do teste e Normal nenhum Atraso ou no máximo um Cuidado; (b) Roteiro de entrevista (Nobre, 2005), que inclui informações sobre a história pré-natal, perinatal e o ambiente familiar; (c) Prontuário Médico dos bebês, que consiste no registro da história de saúde da criança no hospital.

${ }^{1} \mathrm{O}$ presente estudo faz parte da Dissertação de Mestrado da primeira autora sob orientação da última autora. 


\section{Local da Pesquisa}

O estudo foi desenvolvido junto ao Setor de Psicologia Pediátrica (Departamento de Neurociências e Ciências do Comportamento) vinculado ao Ambulatório de Prematuros do Setor de Neonatologia (Departamento de Puericultura e Pediatria) no Ambulatório do Hospital das Clínicas da Faculdade de Medicina de Ribeirão Preto USP, que é um hospital universitário de nível terciário vinculado ao Sistema Único de Saúde (SUS).

\section{Procedimento}

A coleta de dados ocorreu no contexto do Programa de Seguimento Longitudinal de Prematuros (Linhares et al., 2004). Após a alta hospitalar dos bebês nascidos prétermo, as mães são convidadas como rotina a participar do programa de acompanhamento do desenvolvimento das crianças por uma psicóloga treinada e especializada. A inclusão dos bebês e suas respectivas mães no presente estudo ocorreu mediante a assinatura pelas mães do Termo de Consentimento Livre e Esclarecido.

O risco no desenvolvimento dos bebês foi avaliado por meio do Denver-II nas faixas de 5 a 7 meses e 10 a 14 meses de idade cronológica corrigida (ICC). Antes de iniciar a avaliação da criança era identificada a idade cronológica e procedia-se à correção desta, considerando-se a idade gestacional ajustada para 40 semanas, conforme recomendação de Frankenburg et al. (1990). A avaliação do Denver-II era precedida por uma entrevista inicial com a mãe para coletar os dados do ambiente familiar. Ao final do procedimento de avaliação realizavase uma devolutiva para a mãe sobre o desempenho dos bebês com as devidas orientações.

Os dados sobre a história de saúde dos bebês foram coletados dos prontuários médicos, incluindo: peso ao nascimento, idade gestacional, adequação do peso para idade gestacional, escore de Apgar do $5^{\circ}$ minuto, índice de risco clínico neonatal - CRIB (Cockburn et al., 1993) e tempo de internação na UTI Neonatal. O índice CRIB avalia o risco de morbidade e mortalidade dos bebês nas 12 primeiras horas após o nascimento, por meio de seis ítens: peso ao nascimento, idade gestacional, malformação congênita, acidose sanguínea e oxigenação máxima e mínima. A escala varia de zero a 24 pontos e quanto mais alto o escore mais grave o estado do bebê; um índice acima de quatro pontos indica alto risco clínico neonatal (Zardo \& Procianoy, 2003).

O Teste de Denver -II foi corrigido conforme as normas do teste. O desempenho em cada item do teste foi avaliado da seguinte forma: Passou (P), se o bebê conseguisse realizar a tarefa proposta no ítem; Falhou (F), se o bebê não conseguisse realizar a tarefa. No caso de o bebê não ter oportunidade de realizar a tarefa devido às restrições da cuidadora ou por outras razões era marcado. Não houve oportunidade (NO) e no caso de haver recusa em realizar o item por parte do bebê era marcado Recusa (Re). A Falha em cada item deve ser comparada com a amostra do teste e classificada como: Cuidado ( $75 \%$ a $90 \%$ das crianças da faixa etária realizam a tarefa) e Atraso $(90 \%$ ou mais das crianças da faixa etária realizam o item). A classificação de desempenho foi feita de acordo com o número de falhas (Atraso e ou Cuidado). Foi analisado o desempenho total no Denver-II e por áreas separadamente. No tratamento dos dados Normal foi considerado igual a zero e Risco igual a um.

Os dados da entrevista inicial, por sua vez, foram analisados quanto ao conteúdo das respostas dadas pelas mães, com relação às variáveis das fases pré-natal, perinatal e pós-natal e às variáveis do ambiente familiar. As informações obtidas na entrevista e os dados coletados do prontuário médico foram quantificados em termos de frequiência e porcentagem. Após preparação dos dados procedeu-se ao tratamento estatístico utilizando-se o Statistical Package for Social Sciences (SPSS, versão 12.0). Na comparação intra-grupo do desempenho dos bebês nas avaliações do Denver-II, nas faixas etárias de 5 a 7 meses e de 10 a 14 meses (ICC), utilizou-se o teste

Tabela 1

Caracterização da Amostra de Estudo (n=30)

\begin{tabular}{lc}
\hline Características das crianças & Valores \\
\hline Sexo - frequência (porcentagem) & $16(53 \%)$ \\
$\quad$ Feminino & $14(47 \%)$ \\
$\quad$ Masculino & $1.082(660-1.480)$ \\
Peso ao nascimento (gramas) - mediana (mínimo-máximo) & $31(26-34)$ \\
Idade gestacional (semanas) - mediana (mínimo-máximo) & \\
Adequação do peso para idade gestacional ${ }^{(1)}-$ frequêencia (porcentagem) $^{\text {PIG }}$ & $24(80 \%)$ \\
AIG & $6(20 \%)$ \\
Apgar 5 $5^{\circ}$ minuto (escore) - mediana (mínimo-máximo) & $9(5-10)$ \\
Índice de risco clínico neonatal (escore no CRIB & $(2))-$ mediana (mínimo-máximo) \\
Tempo de internação em UTIN (dias) - mediana (mínimo-máximo) & $2(0-10)$ \\
\hline
\end{tabular}

Nota ${ }^{(1)}$ Adequação do peso para idade gestacional: PIG - pequeno para idade gestacional, AIG - adequado para idade gestacional; Segundo classificação de Lubchenco et al. (1966); ${ }^{(2)}$ CRIB - Índice de Risco Clínico Neonatal. 
Nobre, F. D. A., Carvalho, A. E. V., Martinez, F. E. \& Linhares, M. B. M. (2009). Estudo Longitudinal do Desenvolvimento de Crianças Nascidas Pré-Termo no Primeiro Ano Pós-natal.

não-paramétrico de Mc Nemar para amostras pareadas. Procedeu-se à análise do coeficiente de correlação de postos de Spearman para analisar as respectivas relações entre o desempenho no Teste de Denver-II na faixa de 5 a 7 meses e de 10 a 14 meses ICC e as seguintes variáveis: (a) indicadores de risco neonatal (peso ao nascimento, idade gestacional, adequação do peso para idade gestacional, escore de Apgar do $5^{\circ}$ minuto, índice de risco clínico neonatal medido pelo CRIB e tempo de internação na UTIN); (b) sexo; (c) ambiente familiar (escolaridade dos pais, ocupação dos pais, situação conjugal dos pais, número de cômodos, números de pessoas que moram na casa e tipo de moradia).

\section{Resultados}

Observa-se na Tabela 1, que a amostra apresentou uma distribuição semelhante de meninos e meninas (em torno de 50\%). Os bebês apresentaram peso ao nascimento com mediana de 1.082 gramas e idade gestacional mediana de 31 semanas. A maior parte dos bebês apresentou peso ao nascimento abaixo do esperado para idade gestacional (80\%). A mediana do escore do Apgar do $5^{\circ}$ minuto foi alta, de 9 pontos, sugestivo de bom prognóstico e dois pontos no CRIB, o que é sugestivo de baixo risco neonatal para morbidade e mortalidade em sua evolução. Quanto ao tempo de internação na UTIN
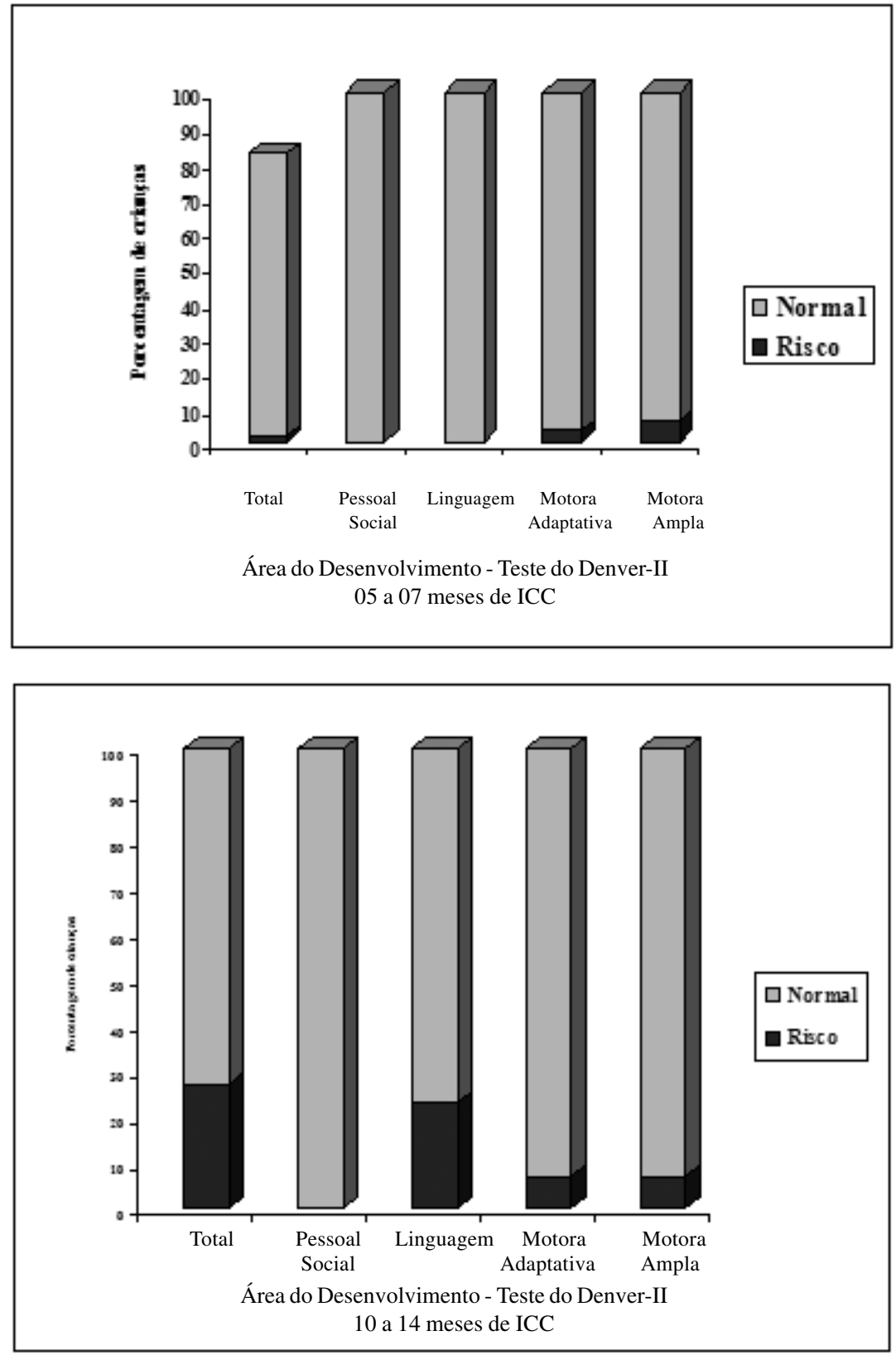

Figura 1. Desempenho no Denver-II das crianças nascidas pré-termo nas faixas de 5 a 7 e 10 a 14 meses de ICC. 
verificou-se que os bebês apresentaram mediana de 25 dias. Observa-se, no entanto, grande amplitude de variação tanto no risco clínico neonatal dos bebês quanto no tempo de internação hospitalar.

Completando esses dados, verificou-se que, com relação às condições pré e peri-natais, a maioria das mães não planejou a gravidez (67\%), contudo as consultas de pré-natal foram realizadas a partir do primeiro trimestre de gravidez (93\%). Pouco mais da metade da amostra de bebês nasceu de parto cesáreo (52\%) e o restante de parto normal (48\%). Com relação ao ambiente familiar, por sua vez, os pais apresentavam na maior parte, baixa escolaridade, sendo que 57\% das mães tinham primeiro grau completo e $29 \%$ segundo grau e $86 \%$ dos pais tinham apenas o primeiro grau completo. A maioria das mães era do lar, sem trabalho remunerado (57\%) e a maior parte dos pais encontrava-se empregado (85\%). Os pais mantinham união estável em $85 \%$ das famílias e viviam em casa própria (50\%), casa alugada (35\%) ou emprestada $(15 \%)$. O número de pessoas residentes na casa variava de duas a sete pessoas (mediana $=4$ ).

Na Figura 1, observa-se que na faixa de 5 a 7 meses ICC houve $20 \%$ de bebês em risco para problemas de desenvolvimento, de acordo com a avaliação do DenverII. A área de desenvolvimento motor apresentou maior porcentagem de bebês em risco em comparação às demais áreas. $\mathrm{Na}$ área Motora ampla incluem-se os itens de controle corporal, sentar, caminhar e pular e, na área Motora adaptativa, os itens de coordenação óculo-manual e manipulação de objetos pequenos. Na faixa de 10 a 14 meses ICC, por sua vez, $27 \%$ dos bebês apresentaram risco para problemas de desenvolvimento no DenverII. Nesta fase o risco foi maior na área da linguagem (23\%) em comparação com as demais áreas avaliadas, envolvendo os itens do tipo: produção de som, capacidade de reconhecer, entender e usar a linguagem para se comunicar.

Tabela 2

Correlação entre Indicadores do Denver-II e Variáveis Neonatais

\begin{tabular}{|c|c|c|c|c|}
\hline $\begin{array}{l}\text { Indicadores do desen- } \\
\text { volvimento (Denver-II) }\end{array}$ & $\begin{array}{l}\text { Faixa etária } \\
\text { (ICC) }\end{array}$ & Variáveis neonatais & $r$ & Valor de $p$ \\
\hline Total & $\begin{array}{l}\text { (5 a } 7 \text { meses }) \\
\text { (10 a } 14 \text { meses) }\end{array}$ & $\begin{array}{l}\text { Risco clínico neonatal - CRIB } \\
\text { Risco clínico neonatal - CRIB } \\
\text { Peso ao nascimento } \\
\text { Tempo de internação na UTIN }\end{array}$ & $\begin{array}{r}0,53 \\
0,39 \\
-0,40 \\
0,49\end{array}$ & $\begin{array}{l}0,004 \\
0,04 \\
0,02 \\
0,01\end{array}$ \\
\hline Área motora ampla & $\begin{array}{l}\text { (5 a } 7 \text { meses }) \\
(10 \text { a } 14 \text { meses })\end{array}$ & $\begin{array}{l}\text { Adequação para idade gestacional (PIG) } \\
\text { Adequação para idade gestacional (PIG) }\end{array}$ & $\begin{array}{l}0,53 \\
0,53\end{array}$ & $\begin{array}{l}0,002 \\
0,002\end{array}$ \\
\hline Área da linguagem & (10 a 14 meses) & $\begin{array}{l}\text { Idade gestacional } \\
\text { Risco clínico neonatal - CRIB } \\
\text { Tempo de internação na UTIN }\end{array}$ & $\begin{array}{r}-0,44 \\
0,41 \\
0,65\end{array}$ & $\begin{array}{l}0,01 \\
0,03 \\
0,001\end{array}$ \\
\hline
\end{tabular}

Nota. ICC $=$ Idade cronológica corrigida.

Comparando-se as duas faixas estudadas, verificou-se que houve diferença estatisticamente significativa entre as fases $(p \leq 0,01)$; os bebês na faixa de 10 a 14 meses ICC apresentaram maior porcentagem de risco na área da linguagem em comparação aos bebês na faixa de 5 a 7 meses ICC. Nas demais áreas não foram observadas diferenças estatisticamente significativas entre as faixas etárias.

Na Tabela 2 nota-se que o resultado total no Denver-II correlacionou-se positivamente com o índice de risco clínico neonatal medido pelo CRIB, nas fases de 5 a 7 e de 10 a 14 meses ICC, negativamente com o peso ao nascimento apenas na faixa de 10 a 14 meses ICC e positivamente com o tempo de internação na UTIN. Portanto, quanto menor o peso ao nascer, maior o risco clínico neonatal e mais tempo de internação na UTIN, maior o risco para problemas de desenvolvimento. Nas áreas específicas do Denver-II foi verificado que os bebês nasci- dos pequenos para idade gestacional apresentaram mais risco no desenvolvimento Motor amplo em ambas as faixas de idade. A área da Linguagem correlacionou-se negativamente com a idade gestacional e positivamente com o índice CRIB e tempo de internação na UTIN; ou seja, os bebês pequenos para idade gestacional, com maior risco clínico neonatal e que ficaram mais tempo internadas na UTIN apresentaram mais risco na área da Linguagem na faixa de 10 a 14 meses ICC.

Complementando os dados da Tabela 2, em relação ao ambiente familiar, verificou-se apenas uma correlação significativa negativa entre o número de pessoas residentes na casa e o desempenho na Linguagem dos bebês na faixa de 10 a 14 meses ICC $(r=-0,38 ; p \leq 0,04)$; os bebês que moravam com maior número de pessoas no lar apresentaram melhor desempenho na linguagem em itens relacionados a combinar sílabas, usar "jargão" e falar "papa" ou "mamã". 
Nobre, F. D. A., Carvalho, A. E. V., Martinez, F. E. \& Linhares, M. B. M. (2009). Estudo Longitudinal do Desenvolvimento de Crianças Nascidas Pré-Termo no Primeiro Ano Pós-natal.

\section{Discussão}

A amostra do presente estudo caracteriza-se por um grupo de bebês com história de risco biológico, nascidos com menos de 34 semanas de idade gestacional (mediana de 31 semanas) e peso abaixo de 1.500 gramas, o que constitui em si fatores de alto risco para o desenvolvimento. Os bebês eram vulneráveis do ponto de vista biológico na fase neonatal e passaram por internação em unidade para tratamento intensivo para assegurar a sua sobrevivência. Porém, observou-se que os bebês estudados apresentaram bom prognóstico de evolução clínica, quando considerados os índices de Apgar do $5^{\circ}$ minuto de vida e de risco clínico neonatal, medido pelo Clinical Risk Index for Babies nas 12 primeiras horas de vida.

A adequada avaliação do risco clínico dos bebês nascidos pré-termo deve levar em conta o conjunto de variáveis biológicas que envolvem o peso ao nascimento e a idade gestacional, assim como os fatores associados de evolução clínica até a alta hospitalar, que melhor caracterizam o nível e tipo da gravidade do risco neonatal desses bebês para problemas de saúde e desenvolvimento (Aywarld, 2002; Linhares et al., 2004).

Paralelamente, além de constituírem-se em um grupo de risco biológico devido ao nascimento prematuro, os bebês pertenciam a famílias com pais de baixa escolaridade, o que representa um relevante fator de risco psicossocial para o desenvolvimento cognitivo de crianças, conforme demonstrado por Andrade et al. (2005). Nesse sentido, a amostra do presente estudo tinha uma condição de múltiplo risco, com a combinação de fatores biológicos e psicossociais.

A avaliação do desenvolvimento inicial dos bebês, para detecção de risco pelo Denver-II mostrou que houve um predomínio de desempenho classificado como Normal em $80 \%$ e $73 \%$ dos bebês da amostra estudada nas faixas de 5 a 7 e de 10 a 14 meses de idade cronológica corrigida, respectivamente. Portanto, o risco relativo para problemas de desenvolvimento foi de $20 \%$ e $27 \%$ em cada uma das faixas estudadas no primeiro ano de vida. Os índices do presente estudo foram menores do que o encontrado por Halpern, Giugliani, Victora, Barros e Horta (2002) em uma amostra de crianças sem risco estabelecido. Esses autores identificaram a presença de $34 \%$ de risco no Denver-II em 1.363 crianças aos 12 meses em crianças que haviam nascido em um hospital de Pelotas e residiam na zona urbana. Além disso, verificou-se neste estudo que a chance de risco aumentava de acordo com a condição de baixo peso, idade gestacional e pobreza, assim como de escolaridade materna, entre outros fatores. Em uma coorte estudada em 2004 pelos mesmos autores foi encontrado um índice de risco de $21,4 \%$, avaliado pelo Denver-II, em crianças nascidas em Pelotas (Halpern et al., 2008); este índice foi próximo ao índice de risco para problemas de desenvolvimento observado no presente estudo.
Na comparação longitudinal do desempenho no Denver-II dos bebês nascidos pré-termo de muito baixo peso do presente estudo, avaliados nas faixas de 5 a 7 e de 10 a 14 meses de idade cronológica corrigida, foi observada uma diferença estatisticamente significativa apenas na área da Linguagem. Isso revela que os bebês apresentaram mais risco para problemas da Linguagem em torno do final do primeiro ano de vida do que na faixa em torno dos seis meses de idade cronológica corrigida. Devese destacar que os itens de linguagem avaliados eram adaptados ao nível de desenvolvimento esperado para cada uma das idades.

Verificou-se, na amostra estudada, que as variáveis neonatais foram associadas ao maior risco no desempenho dos bebês, principalmente no início do desenvolvimento. Os bebês com menor peso ao nascimento, menor idade gestacional, nascidos pequenos para idade gestacional e com alto índice de risco neonatal, apresentaram risco no desenvolvimento avaliado por meio do Denver II.

As variáveis biológicas relacionadas a peso e idade gestacional adequada, por ocasião do nascimento, colaboram para o desenvolvimento sadio, pois exercem papel importante no funcionamento do organismo dos bebês. Contudo, quando esses fatores encontram-se fora dos parâmetros normais esperados, caracterizam-se como fatores de risco para o desenvolvimento típico (Linhares et al., 2004).

Os aspectos do ambiente familiar apresentam importância no desenvolvimento dos bebês nascidos pré-termo. A variável, número de pessoas que residiam com o bebê mostrou relação com o desenvolvimento da linguagem. Os bebês que residiam com maior número de pessoas no lar (em média 4 pessoas) apresentaram menor risco na área da Linguagem avaliada no Denver-II no primeiro ano de vida. Esse achado sugere que a presença de pessoas no lar pode oferecer estimulação adequada, com mediação de estímulos seletivos de aspectos relevantes para mediar a linguagem expressiva dos bebês. Esses achados estão de acordo com o estudo realizado por Schreiber, Effgen e Palisano (1995), ao observar que na medida em que os recursos familiares aumentavam, os pais tornavam-se mais aptos a dar atenção e a investir em seus filhos, seguindo as recomendações de profissionais das áreas de Saúde e de Educação.

Concluindo, os achados do presente estudo mostram que os bebês com baixo peso ao nascimento, menor idade gestacional, maior índice de risco clínico e que ficaram mais tempo de internação na UTI Neonatal têm mais chances de apresentar risco no seu desenvolvimento. Nesse sentido, esses bebês devem ser o alvo das intervenções preventivas nos programas de "follow-up" de prematuros.

Como desdobramento, na prática profissional, os achados do presente estudo reforçam a importância de se avaliar e acompanhar longitudinalmente o desenvol- 
vimento de bebês nascidos pré-termo com muito baixo peso egressas de UTIN, a fim de minimizar riscos e oferecer às famílias orientações necessárias sobre o cuidado com seus filhos. A avaliação sistemática pode possibilitar a identificação de possíveis riscos para o desenvolvimento típico e, conseqüentemente, tal prática pode possibilitar o acompanhamento e a realização da intervenção. Segundo Victora et al. (1996) o diagnóstico inicial de atrasos no desenvolvimento infantil é essencial para melhorar o prognóstico das crianças afetadas.

O acompanhamento longitudinal, de crianças com suspeita de risco para o desenvolvimento torna-se fundamental, segundo Oliveira, Lima e Gonçalves (2003), pois os primeiros anos de vida representam a grande transição na evolução da espécie humana, quando se processam as mais importantes modificações e os maiores saltos evolutivos em um período curto de tempo.

Estudos com delineamento longitudinal, em que os bebês são acompanhados desde o nascimento, têm mostrado grande importância, principalmente quando se referem a avaliações de crianças nascidas prematuras, as quais apresentam alto risco para problemas de desenvolvimento (Linhares et al., 2004)

Cabe ressaltar algumas limitações do presente estudo. Foram encontradas dificuldades na realização do estudo longitudinal, devido ao número de faltas das crianças às consultas de acompanhamento no Ambulatório de Psicologia Pediátrica nas idades selecionadas. Com isso, houve limitação no número de participantes da amostra inicial. Outra limitação do estudo diz respeito à falta da análise de regressão hierárquica, a fim de avaliar o peso das variáveis para explicar os riscos para problemas de desenvolvimento. Devido ao pequeno tamanho da amostra e ao grande número de variáveis, não se realizou tal tipo de análise. Os indicadores de desenvolvimento foram correlacionados às variáveis neonatais e ambientais, o que também não informa sobre o peso e a interação entre as variáveis.

Em relação ao Teste de Denver-II foi de fácil aplicação e cumpriu o objetivo de mensurar o risco para problemas de desenvolvimento infantil. No entanto deve-se ter cautela nas implicações dos resultados, pois o resultado sobre o risco tem apenas a função de alertar para possível risco em potencial. $\mathrm{O}$ teste não tem caráter diagnóstico, mas sim de triagem para identificar de risco para problemas de desenvolvimento das crianças.

O estudo avança no modelo de avaliação e análise proposto que inclui a triagem para problemas de desenvolvimento combinada com a análise dos prontuários médicos dos bebês, o que permitiu de forma adequada um "mapeamento" de indicadores de risco em crianças vulneráveis devido à condição de prematuridade e muito baixo peso ao nascimento.

É de extrema relevância destacar que este estudo foi realizado em um contexto de hospital universitário de nível terciário, a partir da classificação do SUS. Portanto os bebês foram acompanhados por uma equipe multidisciplinar de profissionais da saúde. Dessa maneira, deve-se ter cuidado na generalização dos dados da presente pesquisa para outras amostras de crianças que não se encontram inscritas em programas de "followup", pois estas podem apresentar índices de riscos mais altos em relação os bebês estudados. Por outro lado, o fato do estudo ter sido realizado neste contexto, apresenta "validade ecológica", ao ser realizada a coleta de dados em contexto da realidade de atendimento dos bebês.

Os resultados do estudo remetem a questões importantes para investigações futuras para acompanhamento sistemático de bebês pré-termo com muito baixo peso a fim de investigar possíveis seqüelas no desenvolvimento típico e o enfrentamento das tarefas evolutivas das fases pré-escolar e escolar por meio de estudos com delineamento longitudinal. Outra questão importante diz respeito à análise de interação entre os bebês e as mães, para melhor analisar os indicadores de risco e proteção para o desenvolvimento.

\section{Referências}

Andrade, S. A., Santos, D. N., Bastos, A. C., Pedromônico, M. R. M., Almeida-Filho, N., \& Barreto, M. A. (2005). Ambiente familiar e desenvolvimento cognitivo infantil: Uma abordagem epidemiológica. Revista de Saúde Pública, 39(4), 606-611.

Aywarld, G. P. (2002). Methodological issues in outcomes studies of at- risk infants. Journal of Pediatric Psychology, 27(10), 37-45.

Bordin, M. B. M. (2005). Desenvolvimento psicológico na fase escolar de crianças ex-prematuras, diferenciadas quanto ao risco clínico neonatal. Tese de Doutorado não-publicada, Faculdade de Filosofia Ciência e Letras, Universidade de São Paulo, Ribeirão Preto, SP.

Carvalho, A. E. V., Linhares, M. B. M., \& Martinez, F. E. (2001). História de desenvolvimento e comportamento de crianças prematuras e de baixo peso $(<1500 \mathrm{~g})$. Psicologia: Reflexão e Crítica, 14(1), 1-33.

Cockburn, F., Cooke, R. W. I., Gamsu, H. R., Greenouch, A., Hopkins, A., Macintosh, N., et al. (1993). The CRIB (Clinical Risk Index for Babies) score a tool for assessing initial neonatal risk and comparing performance of Neonatal Intensive Care Units. Lancet, 342, 193-198.

Couperus, J. W., \& Nelson, C. A. (2006). Early brain development and plasticity. In K. Mc Cartney \& D. Phillips (Eds.), Blackwell handbook of early childhood development (pp. 85-105). Portland, OR: Blackwell.

Drachler, M. L., Marshall, T., \& Leite, J. C. C. (2007). A continuous-scale measure of child development for population-based epidemiological surveys: A preliminar study using item response theory for Denver Test. Paediatrics Perinatal Epidemiology, 21, 138-156.

Figueiras, A., Pedromônico, M., Sales, L., \& Figueiras, S. (2000). Manual para vigilância do desenvolvimento da criança de 0 a 2 anos de idade na atenção primária à saúde. Belém, Pará: Secretaria Municipal de Saúde de Belém. 
Nobre, F. D. A., Carvalho, A. E. V., Martinez, F. E. \& Linhares, M. B. M. (2009). Estudo Longitudinal do Desenvolvimento de Crianças Nascidas Pré-Termo no Primeiro Ano Pós-natal.

Fisberg, M., Pedromônico, M. R., Braga, J. A. P., Ferreira, A. M. A., Pini, C., Campos, S. C. C., et al. (1997). Comparação do desempenho de pré-escolares, mediante Teste de Desenvolvimento de Denver, antes e após intervenção nutricional. Revista Associação Médica Brasileira, 43(2), 99-104.

Frankenburg, W. K., Dodds, J., Archer, P., \& Bresnick, B. (1990). Denver II: Technical Manual and Training Manual. Denver, CO: Denver Developmental Materials.

Greenley, R. N., Taylor, G., Drotar, D., \& Minich, N. M. (2007). Longitudinal relationships between early adolescent family functioning and youth adjustment: An examination of the Moderating role of very low birth weight. Journal of Pediatric Psychology, 32(4), 453-462.

Halpern, R., Barros, A. J. D., Matisajevich, A., Santos, I. S., Victora, C. G., \& Barros, F. C. (2008). Estado de desenvolvimento aos 12 meses de idade de acordo com peso ao nascer e renda familiar: Uma comparação de duas coortes de nascimentos no Brasil. Cadernos de Saúde Pública, 24(3), 444-450.

Halpern, R., Giugliani, E. R. J., Victora, C. G., Barros, F. C., \& Horta, B. L. (2002). Fatores de risco para suspeita de atraso no desenvolvimento neuropsicomotor aos 12 meses de vida. Revista Chilena de Pediatria, 73(5), 529-539.

Laucht, M., Esser, G., \& Schmidt, M. H. (2002). Vulnerability and resilience in the development of children at risk: The role of early mother- child interaction. Revista de Psiquiatria Clínica, 29(1), 20-27.

Lewis, R. J., Duglokinski, E. L., Caputo, L. M., \& Griffin, R. B. (1988). Children at risk for emotional disorders: Risk and resources dimensions. Clinical Psychology Review, 8, 417-440.

Linhares, M. B. M. (2004). Estresse, resiliência e cuidados no desenvolvimento de neonatos de alto risco. In G. E. Mendes, M. A. Almeida, \& L. C. A. Williams (Eds.), Temas em Educação Especial (pp. 315-324). São Carlos, SP: Editora da Universidade Federal de São Carlos.

Linhares, M. B. M, Carvalho, A. E. V., Padovani, F. H. P., Bordin, M. B. Martins, I. M. B., \& Martinez, F. E. (2004). Compreensão do fator de risco da prematuridade sob a ótica desenvolvimental. In E. M. Marturano, M. B. M. Linhares, \& S. R. Loureiro (Eds.), Vulnerabilidade e proteção: Indicadores na trajetória de desenvolvimento do escolar (pp. 11-38). São Paulo, SP: Casa do Psicólogo.

Méio, M. D. B. B., Lopes, C. S., Morsch, D. S., Monteiro, A. P. G., Rocha, S. B., Borges, R. A., et al. (2004). Desenvolvimento cognitivo de crianças prematuras de muito baixo peso na idade pré-escolar. Jornal de Pediatria, 80(6), 495-502.

Ministério da Saúde. (n.d.). Cadernos de informações de saúde. Brasília, DF: Autor. Retrieved August 4, 2008, from http:/ /tabnet.datasus.gov.br/tabdata/cadernos/cadernosmap.htm

Mustard, J. F. (2002). Early child development and the brain: The base for health, learning, and behavior throughout life. In M. E. Young, Investing in our children's future (pp. 2362). Washington, DC: The World Bank.

Nobre, F. D. A. (2005). Indicadores do desenvolvimento de crianças nascidas pré-termo de muito baixo peso nos dois primeiros anos de vida. Dissertação de Mestrado nãopublicada, Faculdade de Filosofia Ciência e Letras, Universidade de São Paulo, Ribeirão Preto, SP.

Oliveira, L. N., Lima, M. C. M. P., \& Gonçalves, V. M. G. (2003). Acompanhamento de lactentes com baixo peso ao nascimento: Aquisição de linguagem. Arquivos de Neuropsiquiatria, 61(3), 802-807.
Santa Maria-Mengel, M. R., \& Linhares, M. B. M. (2007). Risk factors for infant developmental problems. Revista Latino-americana de Enfermagem, 15, 837-842.

Santos, R. S., Araújo, A. P. Q. C., \& Porto, M. A. S. (2008). Diagnóstico precoce de anormalidades no desenvolvimento em prematuros: Instrumento de avaliação. Jornal de Pediatria, 84(4), 289-299.

Schreiber, J., Effgen, S. K., \& Palisano, R. J. (1995). Effectiveness of parental collaboration on compliance with a home program. Pediatric Physical Therapy, 7, 59-64.

Silk, J. S., Vanderbilt-Adriance, E., Shaw, D. S., Forbes, E. E., Whalen, D. J., Ryan, N. D., et al. (2007). Resilience among children and adolescents at risk for depression: Mediation and moderation across social and neurobiological contexts. Development and Psychopathology, 19, 841-865.

Silveira, M. F., Santos, I. S., Barros, A. J. D., Matijasevich, A., Barros, F. C., \& Victora, C. G. (2008). Aumento da prematuridade no Brasil: Revisão de estudos de base populacional. Revista de Saúde Pública, 42(5), 957-964.

Victora, C. G., Barros, F. C., Tomasi, E., \& Halpern, R. (1996). Tendências e diferenciais na saúde materno-infantil: Delineamento e metodologia das coortes de 1982 e 1993 de mães e crianças de Pelotas, RS. Cadernos de Saúde Pública, 12(1), 7-14.

Zardo, M. S., \& Procianoy, R. S. (2003). Comparação entre diferentes escores de risco de mortalidade em unidade de tratamento intensivo neonatal. Revista de Saúde Pública, 37(5), 591-596.
Received 30/10/2008 Accepted 10/02/2009 